\title{
ECCO 15: 34th ESMO Multidisciplinary Congress in Berlin: a step forward to a high-quality global oncology forum
}

\author{
Andrés Cervantes*
}

A few weeks ago a joint ECCO/ESMO meeting was held in Berlin. The European Society for Medical Oncology (ESMO), a membership-based society, and the European CanCer Organisation (ECCO), established as a federation of different oncology societies, carried out for the first time a joint meeting in an attempt to combine their efforts and unite forces to host a cobranded biennial multidisciplinary partnership meeting that will provide the best and most up-to-date scientific data for everyone working in cancer. This joint venture plans also to strengthen initiatives aimed at providing all cancer patients with equal access to high-quality cancer treatment.

The ECCO was formed in September 2007, during the 14th European Cancer Conference (ECCO14) in Barcelona, to replace a previous organisation, the Federation of European Cancer Societies (FECS). It has six founding members: the European Association for Cancer Research (EACR), European Society of Surgical Oncology (ESSO), SIOP Europe - the European Society for Paediatric Oncology, European Oncology Nursing Society (EONS), European Society for Therapeutic Radiology and Oncology (ESTRO) and the European Society for Medical Oncology (ESMO). In addition, there are currently seven full members (EANO, EUSOMA, EORTC, ESGO, ESO, ESOP and EUROSKIN) and seven advisory members (EBMT, ECL, Europa Donna, Europa Uomo, FAC, OECI and UICC).

On the other hand, the ESMO is the leading European non-profit, professional organisation for medical oncology promoting multidisciplinary cancer treatment around the world. ESMO unites medical oncologists, oncology specialists, healthcare professionals, caregivers, patients and policymakers in a global alliance committed to eradicating

*AC is a member of the Steering Committee of the Educational Committee of ESMO and chairs the e-learning and continuing medical education working group.

A. Cervantes (ه)

Department of Hematology and Medical Oncology

Institute of Research "Clínico de Valencia"

University of Valencia

ES-46010 Valencia, Spain

e-mail: andres.cervantes@uv.es

cancer and ensuring equal access to high-quality treatment for all patients. Thanks to its state-of-the-art education and training programmes, ESMO plays an instrumental role in providing the oncology community with the most up-todate scientific research and information available. ESMO is dedicated to educating and supporting oncologists, optimising patient care, disseminating cancer-specific information to the public and advocating patient rights.

The ECCO/ESMO meeting can be considered a great success for many different reasons. The first is that more than 15,000 attendees from more than 60 countries were registered at the meeting, a size not previously seen at European Oncology meetings. The second was the educational programme, covering multiple areas of interest and focusing mainly on a multidisciplinary approach to different neoplastic diseases, but paying attention also to basic science and translational research as a keystone for further developments. But, most importantly, a key feature to guaranty the real success of the meeting was the quality and relevance of the papers presented.

Oncology is certainly changing. Like many issues nowadays, oncology is becoming a globalised matter. The amount of information is overwhelmingly increasing and as professionals, institutions and societies we all need to process and interpret this huge amount of data. Moreover, to present and discuss these new findings, independent forums or congresses organised by professional societies are essential to allow the adequate and timely reporting of recently analysed trials and new research achievements.

Not long ago, all the important findings related to any major undertaking from new drug development to phase III trials were only reported at American meetings. Most European investigators were elaborating on their data and waiting for the ASCO to report the findings there. ASCO was the main worldwide forum for the presentation of any new information and the oncology community considered AS$\mathrm{CO}$ as the only source for relevant new findings that should be applied to routine clinical practice. It is important to acknowledge that ASCO has always offered members and guests a very carefully thought out and high-quality educational and scientific programme, selecting important papers to be presented for the first time at the annual meeting. In recent years there has been a marked growth in the contribution of European investigators. For example, the number 
of excellent European abstracts selected for oral presentation or for poster discussion at ASCO has been increasing. Some of the abstracts selected for the plenary session come from European groups and in some areas like breast, lung, colorectal or head and neck cancer the proportion of papers from this side of the Atlantic is significant. At last year's ASCO meeting, seven out of eight abstracts selected for oral presentation at the non-colorectal cancer session were produced and presented by European authors. A similar trend can be seen in other areas of oncology.

As a logical step, if more and better European oncology research is being produced, a global forum to present and discuss it in a European framework is needed. At previous European Congresses, such as ECCO 14 held in Barcelona in 2007 and ESMO 33 held in Stockholm last year, there was a clear upward trend in the number of first-time presented phase III trials and a great effort was made by both organisations to attract high-quality and relevant papers to make the Congress more interesting to professionals and at the same time to increase attendance.

During the last ECCO/ESMO meeting we saw that this is progressing to a more widespread context. More than 50 late breaking abstracts were included in the programme. Among them, nine reported for the first time the results of phase III randomised trials of special interest for early and advanced breast, colorectal, non-small-cell lung and head and neck cancer. A new standard of care was proposed by some of them. Eight of these trials were European multicentre trials or had international cooperation with European participation. These results included data from more than 13500 patients with breast cancer, more than 6000 with colorectal cancer and more than 1300 with lung cancer. Congress organisers as well as participating investigators are to be congratulated for this major achievement. Attendees at this meeting enjoyed an exciting variety of educational activities and a well selected scientific programme.

The other interpretation of these facts is that world oncology may need more than a single American forum every year. It seems clear that researchers are producing enough information and data to split its presentation into at least two first-class congresses. ECCO and ESMO should be aware that this is a big responsibility and when looking at the future, they, as leading organisations, should accept and make the most of this opportunity. Moreover, we can all, as European oncologists, help to build an open and fruitful space for scientific discussion by sending all our best work to the next ESMO or ECCO/ESMO meetings. We are facing the creation, or perhaps better said the consolidation, of a global and high-quality oncology forum at the European level. This old challenge may now be made a concrete reality. At least, this is the impression I got when I attended the last ECCO 15/ESMO 34 joint meeting in Berlin. 